\title{
An Energy Optimization Algorithm for Mobile Ad Hoc Network
}

\author{
${ }^{1}$ Mohamed Belkheir, ${ }^{2}$ Zeyad Qacem, ${ }^{* 3}$ Merahi Bouziani and ${ }^{4}$ Abderrahmane Ghelamellah \\ Telecommunications and Digital Signal Processing Laboratory, BP. 89, Djillali Liabès University, \\ Sidi Bel Abbès,22000 Algeria
E-mail: ${ }^{1}$ belkheir_m@hotmail.com ${ }^{2}{ }^{2}$ ghww.ze1988@hotmail.com; $;{ }^{3}$ bouzi_mera@hotmail.com;
}

\begin{abstract}
Optimized Link-State Routing (OLSR) protocol presents the advantage of finding a route between two nodes in the network in a very short time, thanks to its proactive scheme, but it can expend a lot of resources selecting the MultiPoint Relays (MPR) and exchanging Topology Control information. We present in this paper a new mechanism for OLSR, aiming to improve energy management in mobile ad hoc network; we are describing a modification in the MPR selection based on the willingness concept, by introducing residual energy of node. It is concluded that this solution decreases significantly the number of dead nodes by always choosing stable nodes with important residual energies as MPRs, which prolong the lifetime and enhance the performance of a mobile ad hoc network.
\end{abstract}

Keywords: Mobile ad hoc, OLSR, Quality of service, network life time, Mobile Ad Hoc Network (MANET)

\author{
* Corresponding address: \\ Merahi Bouziani, \\ Telecommunications and Digital Signal Processing Laboratory, BP 89 Djillali Liabès University of Sidi \\ Bel Abbès, Algeria \\ E-mail: bouzimera@hotmail.com \\ Tell: +21348575412 Fax: +21348575412
}

\section{Introduction}

MANET is a collection of wireless mobile nodes, which dynamically form a temporary network, without using any existing network infrastructure or centralized administration. These are often called infrastructure-less networking since the mobile nodes in the network dynamically establish routing paths between themselves. Most recent works in the domain, aim to enhance MANET performances, due to the multiple problems caused by the wireless transmission constraints, and also the limited resources of mobiles nodes. In order to make the network aware of its status at each moment, nodes need to exchange an important number of information, which results a traffic overload, at network level, and more energy consumption at nodes level.

One of well-known routing protocols for MANETs is OLSR. The OLSR is a proactive routing protocol where the routing table of each mobile node is constructed by periodically performing flooding of broadcast packets. In order to reduce the number of broadcast packets, OLSR uses the idea of multipoint relay (MPR) [1]. Each mobile node selects one-hop neighbor nodes as MPR nodes based on their reachability and degree. Only MPR nodes can forward broadcast packets received from other mobile nodes. So MPR nodes transmit more packets than other mobile nodes. In order to efficiently use the energy resource of each mobile node, we have to select MPR nodes in an efficient way.

Efficient utilization of battery energy is very important because today hosts are powered by battery. The energy efficiency is not intended only to reduce power consumption but also to increase lifetime of the node where network maintains certain performance level [2].

We describe in this paper a new extension of OLSR with a novel energy aware mechanism for the MPR selection; we evaluate this new algorithm, and compare its performance with the original OLSR, where willingness didn't depend on residual energy of nodes.

This paper is organized as follows: Section 2 presents the OLSR protocol and related works. Section 3 discusses the proposed modification on OLSR protocol. Section 4 includes simulation environment scenario used in NS-2 simulator. It also, shows performance comparison of OLSR and modified protocol, based on simulation results. Finally we provide a conclusion to our work. 
International Journal of

Soft Computing And Software Engineering (JSCSE)

e-ISSN: 2251-7545

Vol.2,No.10, 2012

Published online: Oct 25, 2012

DOI: 10.7321/jscse.v2.n10.3

\section{Optimized Link-State Routing (OLSR)}

Three proposed protocols have been accepted as experimental RFCs (Request for comments) by the IETF (The Internet Engineering Task Force). They are classified as shown in figure.1.

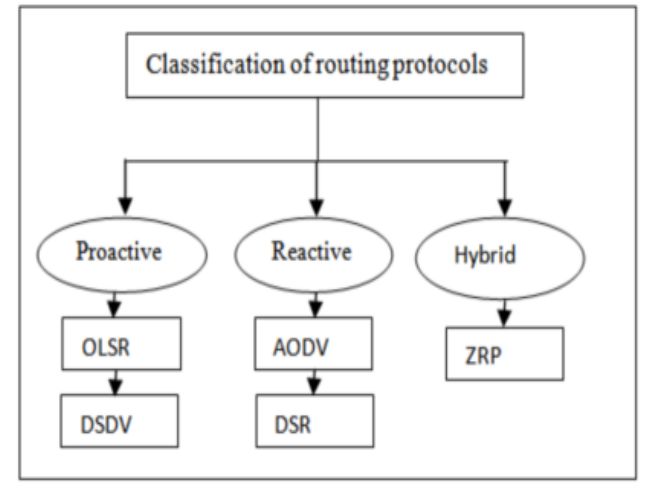

Figure.1. Classification of routing protocols

A proactive approach to MANET routing seeks to maintain a constantly updated topology understanding. The whole network should, in theory, be known to all nodes. This results in a constant overhead of routing traffic, but no initial delay in communication. The proactive characteristic of the protocol provides that the protocol has all the routing information to all participated hosts in the network. Many proactive routing protocols have been proposed, e.g. Destination Sequence Distance Vector (DSDV), Optimized Linked State Routing (OLSR) and so on [3].

Reactive protocols seek to set up routes on-demand. If a node wants to initiate communication with a node to which it has no route, the routing protocol will try to establish such a route. The source node initiates route discovery process by flooding route query within the network. When the destination is reached, route reply request will be sent back to the source. Once the route has been found, it is maintained until either destination becomes inaccessible or the route is no longer desired then route discovery process will be invoked again. Several reactive protocols have been proposed such as Dynamic Source Routing protocol (DSR), ad hoc On-demand Distance Vector (AODV), Temporary Ordered Routing Algorithm (TORA), and so on.

Hybrid protocols seek to combine the proactive and reactive approaches. An example of such a protocol is the Zone Routing Protocol (ZRP); ZRP divides the topology into zones and seeks to utilize different routing protocols within and between the zones based on the weaknesses and strengths of these protocols. ZRP is totally modular, meaning that any routing protocol can be used within and between zones. The size of the zones is defined by a parameter $\boldsymbol{r}$ describing the radius in hops [4].

The OLSR protocol is described in RFC3626. It is a table-driven proactive protocol. As the name suggests, it uses the link-state scheme in an optimized manner to diffuse topology information. In a classic link-state algorithm, link-state information is flooded throughout the network. OLSR uses this approach as well, but since the protocol runs in wireless multi-hop scenarios the message flooding in OLSR is optimized to preserve bandwidth. The optimization is based on a technique called MultiPoint Relaying [1].

The concept of multipoint relaying is to reduce the number of duplicate retransmissions while forwarding a broadcast packet. This technique restricts the set of nodes retransmitting a packet from all nodes, to a subset of all nodes. The size of this subset depends on the topology of the network. This is achieved by selecting neighbors as MPRs. Every node calculates its own set of MPRs as a subset of its symmetric neighbor nodes chosen so that all two-hop neighbors can be reached through a MPR. 
International Journal of

Soft Computing And Software Engineering (JSCSE)

e-ISSN: 2251-7545

Vol.2,No.10, 2012

Published online: Oct 25, 2012

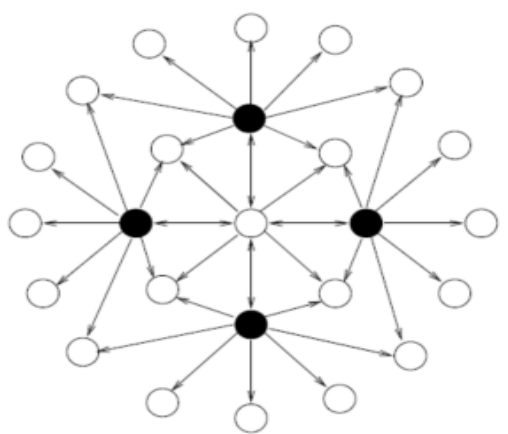

Figure.2. MPR selection in OLSR protocol

The MPR selection, as shown by figure.2, is done by the Hello messages which discover one-hop neighbors as well as two-hop neighbors. Hello messages are broadcast at regular interval (Hello_interval).

Each node of the network maintains topological information about the network obtained with help of TC messages. Each node selected as MPR, broadcast TC message at regular interval (TC_interval). The TC message originated from node which declares MPR selectors of that node.

The neighbor information and the topology information are refreshed periodically, and they enable each node to compute the route to all known destination. These routes are computed with Dijkstr's shortest path algorithm [5]. The Figures 3 and 4 show Hello and TC formats.

\begin{tabular}{|c|c|c|c|}
\hline \multicolumn{2}{|c|}{ Reserved } & Htime & Willingness \\
\hline Link Code & Reserved & Link Message size \\
\hline \multicolumn{2}{|c|}{ Neighbor Interface Address } \\
\hline \multicolumn{2}{|c|}{ Neighbor Interface Address } \\
\hline
\end{tabular}

Figure.3. OLSR Hello Packet Format

The reserved portion in HELLO packets is used for further modification. Htime specifies time before transmission of next HELLO packet. Willingness entry specifies nodes willingness to forward traffic. Link code gives the information about link between sender node and neighbor node. Neighbor interface addressed node address of interface of neighbor node. Link message size gives total length of link message.

\begin{tabular}{|c|c|}
\hline ANSN & Reserved \\
\hline Advertised Neighbor Main Address \\
\hline Advertised Neighbor Main Address \\
\hline$\ldots \ldots \ldots \ldots . .$. \\
\hline
\end{tabular}

Figure.4. OLSR TC Packet Format

Advertised Neighbor Sequence Number (ANSN) which increments sequence number whenever there is change in neighbor set. Advertised Neighbor Main Address field contains main address of neighbor node.

There are various ways to save energy from physical layer to application layer. In network layer energy saving can be possible by selecting energy efficient path for sending data from source to destination. The surveys of different energy saving techniques are discussed as below [3].

C. K. Toh [6] proposed MTPR (Minimum Total Transmission Power Routing), a mechanism which uses a simple energy metric, represented by the total energy consumed to forward the information along 
the route. This way, MTPR reduces the overall transmission power consumed per packet, but it does not affect directly the lifetime of each node (because it does not take account of the available energy of network nodes). Notice that, in a fixed transmission power context, this metric corresponds to a Shortest Path routing.

CMMBCR (Conditional Max Min Battery Capacity Routing) mechanism considers both the total transmission energy consumption of routes and the remaining power of nodes [7]. When all nodes in some possible routes have sufficient remaining battery capacity, i.e. above a threshold, route with minimum total transmission power among the routes is chosen. Since less total power is required to forward packets for each connection, the load for most of the nodes must be reduced, and thereby lifetime will be extended. But, if all routes have nodes with low battery capacity i.e. below the defined threshold, a route including nodes with lowest battery capacity must be avoided to extend the lifetime of these nodes [3].

\section{Optimized Energy OLSR Protocol (OE-OLSR)}

In this section, we are going to discuss the modification steps for OLSR protocol. The modified protocol is named OE-OLSR.

OE-OLSR protocol optimizes the energy consumption over the network as well as increases the network lifetime. We propose to select the nodes which have high residual energy as MPR, and by avoiding the nodes with minimum battery lifetime.

Authors presented many ways to make routing protocol, aware about mobility or energy, in many works, authors choose to make nodes exchanging their information about a number of parameters, so that every node can construct its proper repository based on data collected from its neighbors, this approach has several advantages, each node can have a local idea about the networks, and can use those information's to build certain specific route with specific constraints, but produces a supplement traffic [5]. In our approach, Unless MPRs are used to construct route from any source to any destination in the network, we choose to bind those parameters to willingness, each node must decide about his ability to became an MPR, based on his residual energy, this proposition has the advantage to be compatible with the standard and need no more control traffic or modifications of the core functioning of OLSR.

The existing OLSR protocol doesn't take care of energy constraints which results in short network lifetime. To improve network lifetime as well as energy efficiency, the OLSR was modified by setting two thresholds for residual energy.

For MPR selection, we have decided two thresholds $80 \%$ and $10 \%$ :

- If the residual energy is greater than $80 \%$, then node having HIGH-MPR-WILL

- If the residual energy is less than $10 \%$, then node will be declared as rationally dead. The node which is marked as rationally dead can only receive packets transmitted at it, it can never been selected to forward packets to other nodes.

HELLO messages are used for selection of MPR nodes. For selection of MPR each node having:

- Highest residual energy

- Lowest residual energy

- Default residual energy

The objective of applying this technique is to minimize the total power consumption by avoiding nodes with minimum battery lifetime as well as increase the lifetime of the network.

There is need to update residual energy of each node at regular interval. For this purpose the value of residual energy by node is included in HELLO packet as shown in figure.5. Each node sends HELLO packet with entry for current residual energy and depending on threshold value set to select MPR node. 
International Journal of

Soft Computing And Software Engineering (JSCSE)

e-ISSN: 2251-7545

Vol.2,No.10, 2012

Published online: Oct 25, 2012

\begin{tabular}{|l|l|l|l|}
\hline Reserved & $\begin{array}{l}\text { Residual } \\
\text { Energy }\end{array}$ & Htime & Willingness \\
\hline Link code & Reserved & Link message size \\
\hline \multicolumn{3}{|c|}{ Neighbors interface adresse } \\
\hline \multicolumn{3}{|c|}{ Neighbors interface adresse } \\
\hline
\end{tabular}

Figure.5. New Hello packet format

The reserved part in HELLO packet is modified with entry for residual energy of node.

The reserved part in TC packet, as shown in figure.6, is modified also with entry for residual energy of node. The TC packets are used to disseminate topology information over entire network. The modified TC packet format distributes residual energy of each node over entire network. After knowing topology information for each node in network the route calculation is performed.

\begin{tabular}{|l|l|l|}
\hline ANSN & Residual Energy & Reserved \\
\hline Advertised Neighbbours Main Address \\
\hline \multicolumn{3}{|c|}{ Advertised Neighbbours Main Address } \\
\hline \multicolumn{3}{|c|}{$\ldots \ldots \ldots \ldots \ldots \ldots \ldots$} \\
\hline
\end{tabular}

Figure.6. New TC packet format

\section{Simulation Environment}

We use the metric "number of dead nodes" in our simulation trials. This metric indicates the overall lifetime of the network [8]. In wireless Ad Hoc networks, especially in those with densely distributed nodes, the death of the first node seldom leads to the total failure of the network. With number of dead nodes increasing, the network is likely to partition. More importantly, it gives an idea of the area coverage of the network over time. Network lifetime is one of important metrics to evaluate the energy efficiency of the routing protocols with respect to network partition [9].

The MANET network simulations are implemented using NS-2 simulator. Nodes in the simulation move according to Random Waypoint Mobility model [10]. Each node is then assigned a particular trajectory. The simulation period for each scenario is 100 seconds. The MAC layer protocol IEEE 802.11 is used in all simulations with the data rate 2 packets/s. The application used the Constant Bit rate (CBR) traffic to generate packets. Table. 1 presents all simulation parameters.

Table.1. Simulation parameters

\begin{tabular}{|l|l|l|l|}
\hline Parameters & Number of nodes & Node speed & Number of connections \\
\hline Area & $870 \times 870$ & $870 \times 870$ & $870 \times 870$ \\
\hline Nodes & $\mathbf{2 0 , 4 0 , 6 0 , 8 0 , 1 0 0}$ & 40 & 40 \\
\hline Node Speed & $3 \mathrm{~m} / \mathrm{S}$ & $\mathbf{1 , 3 , 5 , 8 , 1 0}$ & $3 \mathrm{~m} / \mathrm{S}$ \\
\hline Simulation time & $100 \mathrm{~s}$ & $100 \mathrm{~s}$ & $100 \mathrm{~s}$ \\
\hline Traffic Type & CBR & CBR & CBR \\
\hline Traffic source & $9,18,27,36,45$ & 16 & $\mathbf{1 0 , 1 2 , 1 4 , 1 6 , 1 8}$ \\
\hline Packet rate & 2 packets & 2 packets & 2 packets \\
\hline Initial power & 100 joules & 100 joules & 100 joules \\
\hline Routing protocol & OLSR, OE-OLSR & $\begin{array}{c}\text { OLSR,OE- } \\
\text { OLSR }\end{array}$ & OLSR, OE-OLSR \\
\hline
\end{tabular}

\section{Simulation Results}

We are studying in this section the performance extension of OLSR: Optimized Energy-OLSR , where decision about willingness depends on residual energy of node, we compare this extension with the original version of OLSR.

We evaluate essential Quality of Service parameters to analyze the performance differences of OLSR and OE-OLSR. Each node in the network has some constant Initial energy (100 joules). The QoS parameter, "dead nodes", is chosen to show that less number of nodes is dead for longer time in the network. More number of alive nodes implies the optimization of energy [11]- [14].

Figure.7 Shows effects of speed, number of nodes and connection number on number of dead nodes. In case of OE-OLSR number of dead nodes has a lesser value than OLSR. 


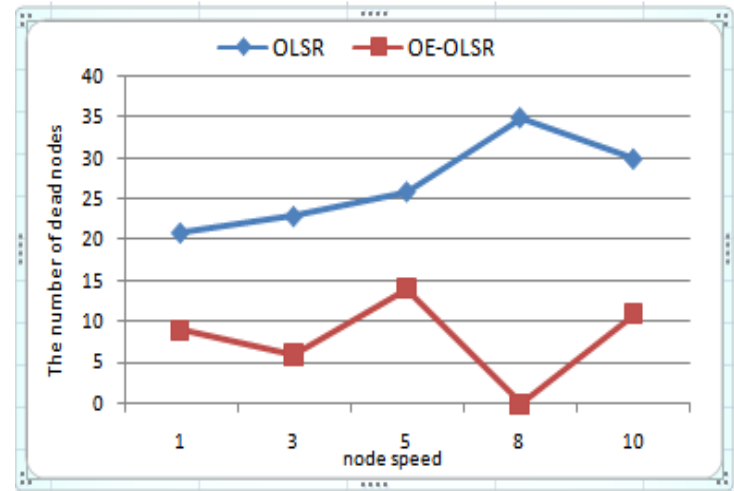

(a)

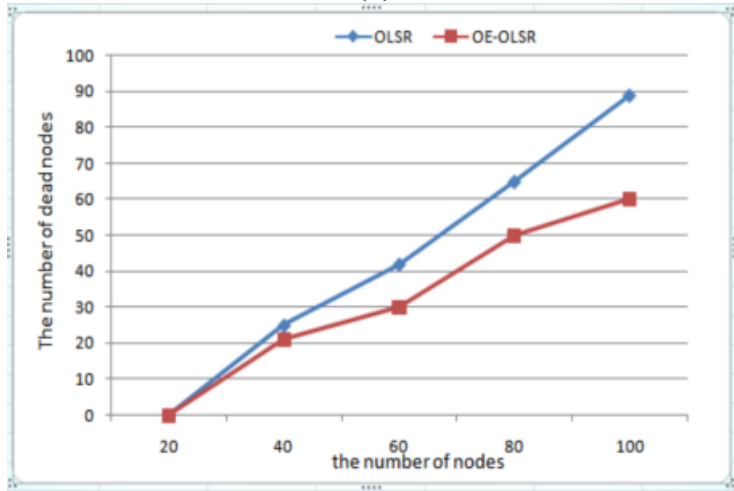

(b)

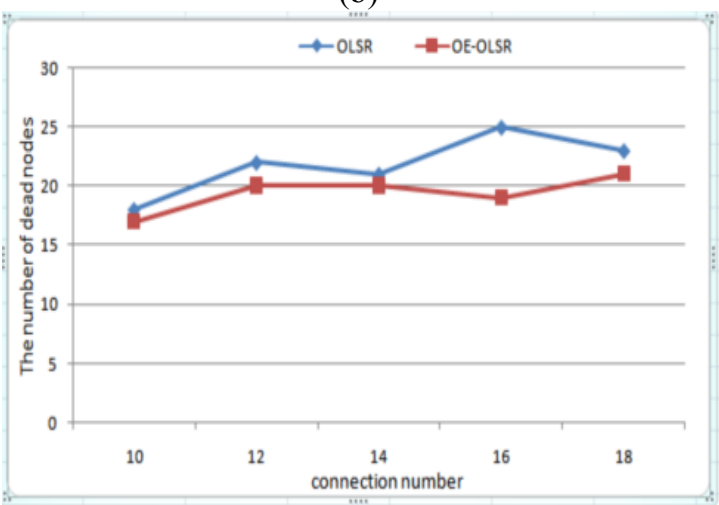

(c)

Figure.7. Number of dead nodes vs (a): node speed, (b) number of nodes, (c) number of connection

As shown by Figure.7, OE-OLSR enhances network performance, nodes with low level of battery power will not be able to set themselves as MPRs, which give them the opportunity to preserve their energy and continue to send and receive packets for a significant time compared with OLSR.

By varying number of nodes, it has been observed that OE-OLSR has less number of nodes dead, for high node density. So it can be seen from the results, OE-OLSR is best suitable for dynamic and dense network.

Obviously, it is seen that for the three metrics there is an increasing trend of number of dead nodes, which results in prolonging of networks' lifetime.

\section{Conclusion}

In this paper, we compared our protocol OE-OLSR over OLSR with help of performance metrics such as the number of dead nodes as QoS parameter. OLSR always uses shortest hop route, so congestion occurs and distribution of load is not considered. Also, OLSR does not consider available node energy of 
International Journal of

Soft Computing And Software Engineering (JSCSE)

e-ISSN: 2251-7545

Vol.2,No.10, 2012

Published online: Oct 25, 2012

nodes for path selection and communication purposes. The proposed protocol combines the features of existing techniques to decrease the energy consumption and increase the lifetime of nodes and network.

Our simulation results demonstrate that the proposed OE-OLSR protocol outperforms OLSR for number of dead nodes after such time; as well as increasing the lifetime of the network. Future work will to choose the residual energy thresholds depending on the number of dead nodes.

\section{References:}

[1] F. Lakrami, N. Elkamoun, "Energy and mobility in OLSR routing protocol", Cyber Journals: Multidisciplinary Journals in Science and Technology, Journal of Selected Areas in Telecommunications (JSAT), March Edition, 2012

[2] Change J-H, Tassilas L. "Energy Conserving Routing in wireless ad hoc networks", proceedings of the conf. on Computer Communications (IEEE Infocom), 22-31, 2000

[3] Zhan Huawei, Zhou Yun "Comparison and Analysis AODV and OLSR Routing Protocols in Ad Hoc Network", IEEE Transactions, pp. 1-4, 2008

[4] S. Gowrishankar, T.G. Basavaraju, M. Singh, Subir Kumar Sarkar, "Scenario based Performance Analysis of AODV and OLSR in Mobile Ad hoc Networks", Proceedings of the 24th South East Asia Regional Computer Conference, November 18-19, 2007, Bangkok, Thailand.

[5] Marco Fotino, Salvatore Marano "EE-OLSR: Energy Efficient OLSR Routing Protocol for Mobile Ad hoc Networks", IEEE transactions, pp.1-7, 2008

[6] C.-K. Toh "Maximum Battery Life Routing to Support Ubiquitous Mobile Computing in Wireless Ad Hoc Networks", in IEEE Communication Magazine, June 2001.

[7] Mayur Tokekar, Radhika D. Joshi "Extension of optimized Linked State Routing Protocol for Energy Efficient”, International Journal on Ad Hoc Networking Systems (IJANS) Vol. 1, No. 2, October 2011.

[8] Arati Manjeshwar and Dharma P. Agrawal, (2001) "TEEN: A Routing Protocol for Enhanced Efficiency in Wireless Sensor Networks", Fifteenth International Parallel and Distributed Processing Symposium (IPDPS'01) Workshops, Vol. 3, pp. 30189a, 2001.

[9] S. Lindsey, K. Sivalingam, C. S. Raghavendra, "Power Optimization in Routing Protocols for Wireless and Mobile Networks," Handbook of Wireless Networks and Mobile Computing, I. Stojmenovic, Ed., Wiley, 2001.

[10] Tracy Camp, Jeff Boleng and Vanessa Davies, "A survey of Mobility Models for Ad hoc Network Research", Wireless Communications and Mobile computing: A special issue on Ad hoc network Research, vol 2, No5, pp. 483-502, 2002.

[11] Thomas Kunz "Energy-Efficient Variations of OLSR", IEEE Transactions, pp. 517-522, 2008

[12] Jamal Toutouh et Enrique Alba, «An efficient routing protocol for green communications in vehicular ad-hoc networks », dans GECCO '11 Proceedings of the 13th annual conference companion on Genetic and evolutionary computation, ACM Press, p.719-725, 2011

[13] Saoucene Mahfoudh et Pascale Minet, «Energy-aware routing in wireless ad hoc and sensor networks », dans Proceedings of the 6th International Wireless Communications and Mobile Computing Conference, ACM Press, p. 1126-1130, 2010

[14] Kirti Aniruddha Adoni and Radhika D. Joshi, «Optimization of Energy Consumption for OLSR Routing Protocol in MANET», International Journal of Wireless \& Mobile Networks (IJWMN) Vol. 4, No. 1, p. 251-262, February 2012 